\title{
Endoplasmic reticulum stress associated apoptosis as a novel mechanism in indoxyl sulfate-induced cardiomyocyte toxicity
}

\author{
XIAO TAN $^{1 *}$, XUE-SEN CAO $^{2-4 *}$, PAN ZHANG $^{1}$, FANG-FANG XIANG $^{2-4}$, \\ JIE TENG $^{2,3}$, JIAN-ZHOU ZOU ${ }^{2-4}$ and XIAO-QIANG DING ${ }^{1-5}$ \\ ${ }^{1}$ Shanghai Key Laboratory of Kidney and Blood Purification; ${ }^{2}$ Department of Nephrology, \\ Zhongshan Hospital, Fudan University; ${ }^{3}$ Shanghai Institute of Kidney and Dialysis; \\ ${ }^{4}$ Hemodialysis Quality Control Center of Shanghai; ${ }^{5}$ Shanghai Medical Center of Kidney Disease, \\ Shanghai 200032, P.R. China
}

Received January 28, 2018; Accepted July 5, 2018

DOI: $10.3892 / \mathrm{mmr} .2018 .9496$

\begin{abstract}
Indoxyl sulfate (IS), a typical uremic toxin, is of great importance in the development of chronic kidney disease. In addition to its nephrotoxicity, previous studies have provided increasing evidence for its cardiovascular toxicity. The mechanism underlying IS-induced cardiovascular toxicity has been elusive to date. The present study aimed to evaluate whether IS treatment could induce apoptosis of H9C2 cells, and used the endoplasmic reticulum (ER) stress-modulator 4-phenylbutyric acid (4-PBA) to evaluate whether IS-induced apoptosis is indeed associated with ERS. To evaluate whether IS induces apoptosis in H9C2 cardiomyocytes, cells were exposed to increasing concentrations of IS $(500,1,000$, and 2,000 $\mu \mathrm{M})$ for $24 \mathrm{~h}$, and apoptosis was detected by flow cytometry. To determine whether IS-induced apoptosis is associated with ERS, cells were divided into 4 groups: Control group, PBA group, IS group and PBA+IS group. IS dose-dependently induced apoptosis, and increased the expression of ER chaperones in H9C2 cells. Additionally, 4-PBA treatment decreased IS-induced apoptosis, and reduced ERS-associated protein expression induced by IS. Therefore, the mechanism may be associated with the CCAAT-enhancer-binding protein homologous protein and c-Jun N-terminal kinase signaling pathways.
\end{abstract}

Correspondence to: Professor Xiao-Qiang Ding or Dr Jian-Zhou Zou, Department of Nephrology, Zhongshan Hospital, Fudan University, 180 Fenglin Road, Shanghai 200032, P.R. China

E-mail: ding.xiaoqiang@zs-hospital.sh.cn

E-mail: zou.jianzhou@zs-hospital.sh.cn

${ }^{*}$ Contributed equally

Key words: uremic toxin, indoxyl sulfate, cardiomyocyte, apoptosis, endoplasmic reticulum stress

\section{Introduction}

Cardiovascular disease remains the leading cause of morbidity and mortality in patients with chronic kidney disease (CKD), particularly in patients who elect to undergo dialysis treatment $(1,2)$. Indoxyl sulfate (IS) is a typical protein-bound uremic toxin, which is challenging to remove using current dialysis techniques, and serves a central role in cardiac damage among patients with CKD (3). The authors' previous clinical research suggested that IS is associated with heart failure in patients undergoing hemodialysis (4). However, the mechanism underlying IS-induced cardiac tissue damage has yet to be fully revealed. Research has revealed that IS promotes cardiomyocyte hypertrophy and cardiac fibrosis via enhanced oxidative stress (5), and suppresses anti-oxidative mechanisms (6). Studies have also demonstrated that prolonged endoplasmic reticulum (ER) stress may serve an important role in cardiomyocyte hypertrophy and apoptosis (7-9). The ER is the cellular compartment in which proteins are synthesized and acquire the correct three-dimensional configuration needed for biological activity. When cells synthesize secretory proteins in quantities that exceed the capacity of the ER, unfolded proteins accumulate in the ER, inducing ER stress (ERS). In response to ERS, various ER chaperones, including glucose-regulated protein 78 (GRP78), are upregulated. When ERS is excessive and/or prolonged, the initiation of apoptosis is promoted by the transcriptional induction of CCAAT-enhancer-binding homologous protein (CHOP), and/or by the activation of c-Jun N-terminal kinase (JNK)-dependent pathways (10).

In the current study, the authors sought to examine whether IS causes cell apoptosis, and whether ERS participates in the molecular mechanism underlying IS-induced cardiac toxicity.

\section{Materials and methods}

Cells, culture and reagents. H9C2 cardiomyocytes (Type Culture Collection of the Chinese Academy of Sciences, Shanghai, China) were taken from liquid nitrogen and cultivated in Dulbecco's modified Eagle's medium (DMEM; Gibco; Thermo Fisher Scientific, Inc., Waltham, MA, USA) supplemented with $10 \%$ fetal bovine serum (Gibco; Thermo 
Fisher Scientific, Inc.), and maintained at $37^{\circ} \mathrm{C}$ in a humidified atmosphere containing $5 \% \mathrm{CO}_{2}$. For different experiments, cells were seeded into 6 - $\mathrm{cm}$ dishes or 6-well plates, or onto 12-mm-diameter glass coverslips in 24-well plates. IS and 4-phenylbutyric acid (4-PBA) were purchased from Sigma-Aldrich (Merck KGaA, Darmstadt, Germany).

Experimental groups. To evaluate whether IS induces apoptosis in H9C2 cardiomyocytes, cells were exposed to increasing concentrations of IS $(500,1,000$, and 2,000 $\mu \mathrm{M})$ for $24 \mathrm{~h}$, and apoptosis was detected by flow cytometry. To determine whether IS-induced apoptosis is associated with ERS, cells were divided into 4 groups: i) Control group $(n=4)$ : Cells were treated with serum-free DMEM for $48 \mathrm{~h}$, culture medium was changed after the first $24 \mathrm{~h}$; ii) PBA group $(\mathrm{n}=4)$ : Cells were treated with PBA (1 mM) for $24 \mathrm{~h}$, and then cultured with DMEM for $24 \mathrm{~h}$; iii) IS group $(\mathrm{n}=4)$ : Cells were treated with DMEM for $24 \mathrm{~h}$, and then treated with IS ( $1 \mathrm{mM})$ for $24 \mathrm{~h}$; and iv) PBA+IS group ( $\mathrm{n}=4)$ : Cells were pretreated with PBA $(1 \mathrm{mM})$ for $24 \mathrm{~h}$, prior to IS $(1 \mathrm{mM})$ treatment for $24 \mathrm{~h}$. Serum-free DMEM was used in all experimental groups. The present study determined $1 \mathrm{mM}$ to be the optimal concentration of PBA, according to a previous study (11), and determined $1 \mathrm{mM}$ to be the optimal concentration of IS with respect to its induction of apoptosis and ERS, which is presented in the results.

Flow cytometry. An apoptosis kit with Annexin-V Alexa Fluor $^{\mathrm{TM}} 488$ and propidium iodide (PI; Thermo Fisher Scientific, Inc.) was used to evaluate cell apoptosis. Cells were washed with cold PBS and binding buffer, and then stained with AnnexinV-FITC for $20 \mathrm{~min}$ at $4^{\circ} \mathrm{C}$, followed by PI for 5 min per sample. Data were acquired using an Attune NxT Flow Cytometer (Thermo Fisher Scientific, Inc.), and FlowJo software (v.14.0; TreeStar, Inc. San Carlos, CA, USA) was used for data analysis.

Western blot assay. Protein samples from H9C2 cells were lysed in radio immunoprecipitation assay buffer (catalog no. KGP702, Keygen, Jiangsu, China). Lysates were centrifuged at $12,000 \mathrm{x} \mathrm{g}\left(4^{\circ} \mathrm{C}\right)$ for $5 \mathrm{~min}$. Supernatants were collected, and equal amount of proteins (50 $\mu \mathrm{g}$ per sample) were subjected to $10 \%$ SDS-PAGE, followed by transference to Immobilon-PVDF membranes (Merck KGaA, Darmstadt, Germany) for 90 min at $110 \mathrm{~V}$ on ice. Membranes were incubated in $5 \%$ non-fat milk for $60 \mathrm{~min}$ at room temperature, and then reacted overnight at $4^{\circ} \mathrm{C}$ with specific antibodies: Anti-GRP78 (1:1,000, catalog no. 3183), anti-CHOP (1:1,000, catalog no. 2895), anti-phospho (p)-stress activated protein kinase (SAPK)/JNK (1:1,000, catalog no. 9255), anti-SAPK/JNK (1:1,000, catalog no. 9252), anti-cleaved-caspase 3 (1:1,000, catalog no. 9664) and anti-caspase3 (1:1,000, catalog no. 9662) were purchased from Cell Signaling Technology, Inc. Danvers, MA, USA. The membranes were rinsed in PBS 3 times (10 min each time), then incubated with the peroxidase-conjugated AffiniPure Goat anti-rabbit IgG antibody (1:10,000, catalog no. 111-035-003, Jackson ImmunoResearch Laboratories Inc., PA, USA) and peroxidase-conjugated AffiniPure Goat anti-mouse IgG antibody (1:10,000, catalog no. 115-035-003, Jackson ImmunoResearch Laboratories Inc., PA, USA) for $1 \mathrm{~h}$ at room temperature. Then, the membranes were rinsed 3 times (10 min each time) in PBS. Proteins were visualized by enhanced chemiluminescent reagents (Thermo Scientific, Inc.), and band intensity was analyzed using program Image-J software (v.1.4.3.67; National Center for Biotechnology Information).

Statistical analysis. All experiments were performed at least in triplicate. Data are presented as the mean \pm standard deviation. Statistical differences were determined using analysis of variance coupled with Dunnett test or Tuckey's test. Statistical analysis was performed using SPSS software, version 22.0 (IBM SPSS, Armonk, NY, USA). P<0.05 was considered to indicate a statistically significant difference.

\section{Results}

IS stimulation induces apoptosis and ERS. Treatment of H9C2 cells was conducted using an IS concentration range of $0-2,000 \mu \mathrm{M}$, and cells were collected for apoptosis evaluation. IS (1,000-2,000 $\mu \mathrm{M})$ significantly stimulated both early and late apoptosis (Fig. 1). Treatment of H9C2 cells occurred at IS concentrations ranging from $0-1,000 \mu \mathrm{M}$, and cells were collected for western blot analysis. Compared with the control group, the expression of the ER chaperone GRP78 was significantly increased in the IS $500 \mu \mathrm{M}(\mathrm{P}<0.05)$ and $1,000 \mu \mathrm{M}$ $(\mathrm{P}<0.01)$ treated groups (Fig. 2). IS induced both apoptosis and ERS at a concentration of $1,000 \mu \mathrm{M}$. Therefore, 1,000 $\mu \mathrm{M}$ IS was used for subsequent experiments.

Apoptosis of H9C2 is associated with ERS in the context of IS stimulation. H9C2 cardiomyocytes were divided into 4 groups: i) Control group; ii) 4-PBA group; iii) IS group; and iv) 4-PBA+IS group. Compared with the control group, early apoptosis was significantly increased in the IS group $(\mathrm{P}<0.001)$, and early apoptosis was significantly attenuated in the 4-PBA+IS group, when compared with the IS group $(\mathrm{P}<0.001)$. A similar trend was observed with the late apoptosis phase, as presented in Fig. 3. Compared with the control group, the expression of cleaved-caspase-3 in the IS and 4-PBA+IS groups was significantly increased. Compared with IS group, the expression of cleaved-caspase-3 protein is significantly lower in 4-PBA+IS group (Fig. 4).

JNK-CHOP signaling pathway activity increases under IS stimulation. The protein expression levels of GRP78, CHOP and p-JNK in each group are presented in Fig. 4. Compared with the control group, the expression levels of GRP78 and $\mathrm{CHOP}$ in the IS and 4-PBA+IS groups were significantly increased, whereas the phosphorylation of JNK was only increased in the IS group. Compared with the IS group, the protein expression levels of GRP78 and CHOP were significantly decreased in the 4-PBA+IS group, as was the phosphorylation of JNK. No statistically significant difference was observed in the expression of these proteins between the control and the 4-PBA groups.

\section{Discussion}

The mechanisms of cardiovascular disease in hemodialysis patients are complex. Conventional risk factors, such as hypertension and volume overload, are considered 

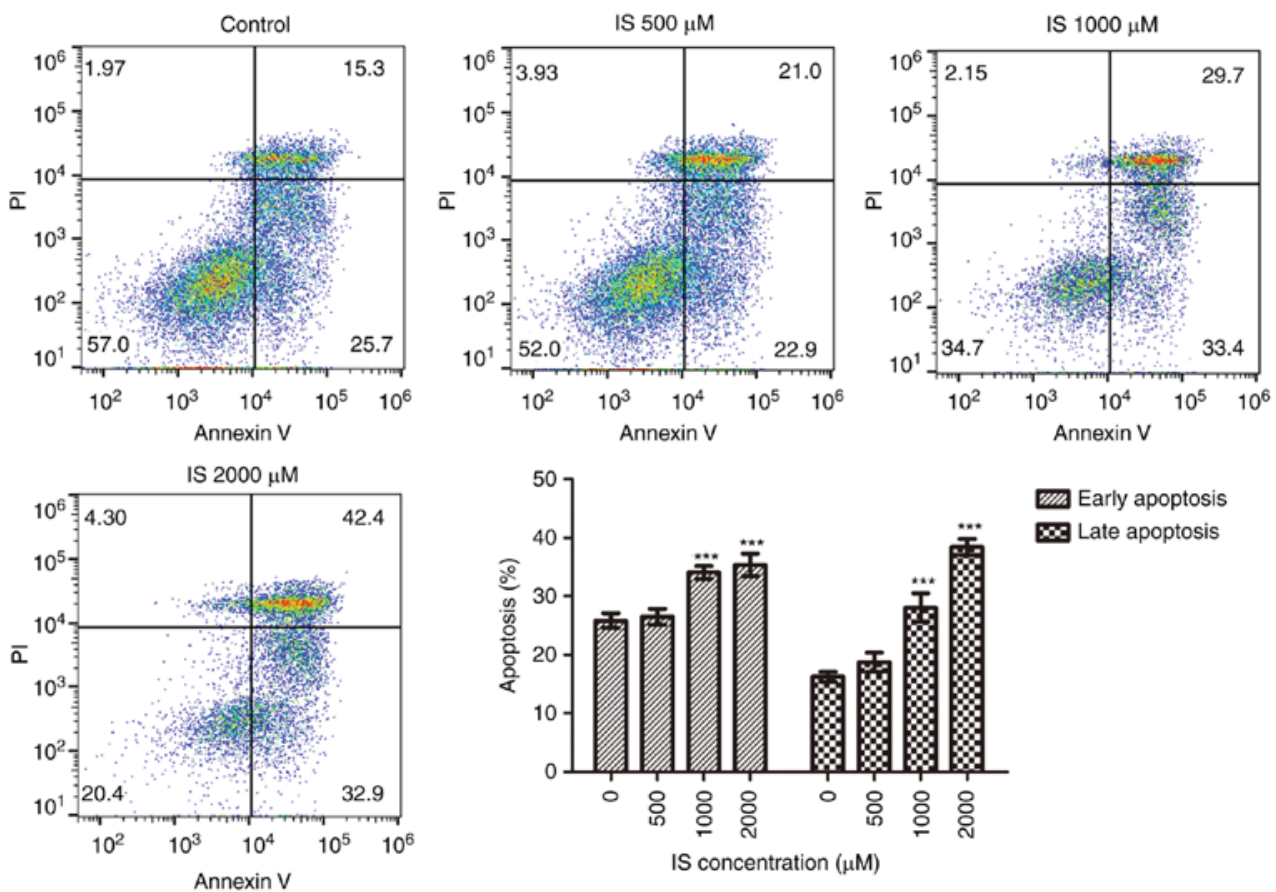

Figure 1. Determination of apoptosis in $\mathrm{H} 9 \mathrm{C} 2$ cells. The apoptosis rate of $\mathrm{H} 9 \mathrm{C} 2$ cells was measured following treatment with different concentrations of IS $(\mathrm{n}=4) .{ }^{* * *} \mathrm{P}<0.001$ vs. control group. IS, indoxyl sulfate; PI, propidium sulfide.
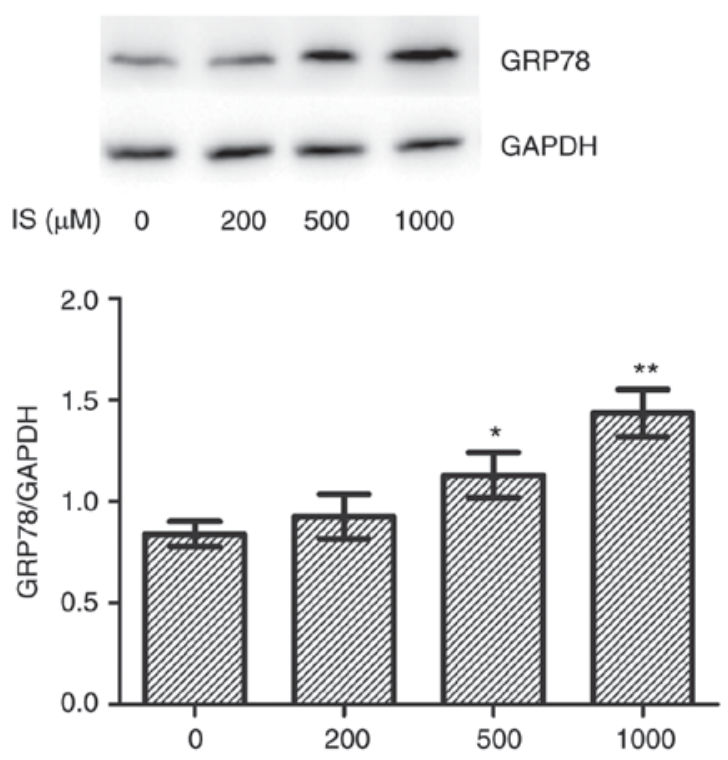

Figure 2. Protein expression level of GRP78. The protein expression level of endoplasmic reticulum chaperone GRP78 was determined in $\mathrm{H} 9 \mathrm{C} 2$ cells following treatment with different concentrations of IS $(n=4) .{ }^{*} \mathrm{P}<0.05$, ${ }^{* * *} \mathrm{P}<0.01$ vs. control group. IS, indoxyl sulfate; GRP78, glucose-regulated protein 78

to play prominent roles in uremic cardiomyopathy (12). However, previous research demonstrated that a reduction of blood pressure has little effect in preventing the development of uremic cardiac hypertrophy (13). The authors hypothesized that non-established risk factors, including uremic toxins, have been underestimated and are important in the progression of cardiovascular disease in CKD. IS is a typical protein-bound uremic toxin that cannot be removed by current hemodialysis techniques, and has been demonstrated to have a critical effect on the prognosis of cardiovascular disease induced by CKD, both in pre-clinical (5,14-16) and clinical (4,17-19) research. The conventional mechanism of pathological cardiac hypertrophy involves $\beta$-adrenergic receptor-, $\alpha$-adrenergic receptor-, and $\mathrm{Ca}^{2+} /$ calmodulin-related pathways, while the key mechanism of IS-induced cardiac hypertrophy is the direct induction of oxidative stress (5). It is important to further investigate the mechanism of IS-induced cardiomyocyte injury, and to explore potential therapeutic targets.

There is plenty of evidence to suggest that the key mechanism of IS cardiotoxicity is the induction of oxidative stress $(5,16,20)$. ERS and oxidative stress are closely related in many physiological and pathological conditions (21). Protein misfolding in ERS conditions results in reactive oxygen species production, and oxidative stress disturbs the ER redox state and promotes protein misfolding (22). Therefore, the authors hypothesized that ERS may also play an important role in the harmful effects of IS. GRP78 is an ER molecular chaperone, and a specific marker of ERS (10). The present study analyzed the rate of apoptosis in $\mathrm{H} 9 \mathrm{C} 2$ cardiomyocytes, and GRP78 protein expression, and it was demonstrated that IS dose-dependently induced ERS, and promoted the apoptosis of H9C2 cardiomyocytes. Previous studies demonstrated that, Indoxyl sulfate induces apoptosis of kidney mesangial cells (23), renal tubular cells $(24,25)$ and osteoblast cells (26), which are in accordance with the present results. Importantly, the study showed that the ERS modulator 4-PBA attenuated the effect of IS on ERS and apoptosis induction, which indicated that the apoptosis induced by IS treatment is likely via ERS induction.

It was further demonstrated that IS increased the protein expression of GRP78, CHOP, and p-JNK, which could be suppressed by the ER inhibitor 4-PBA, and this indicating that 

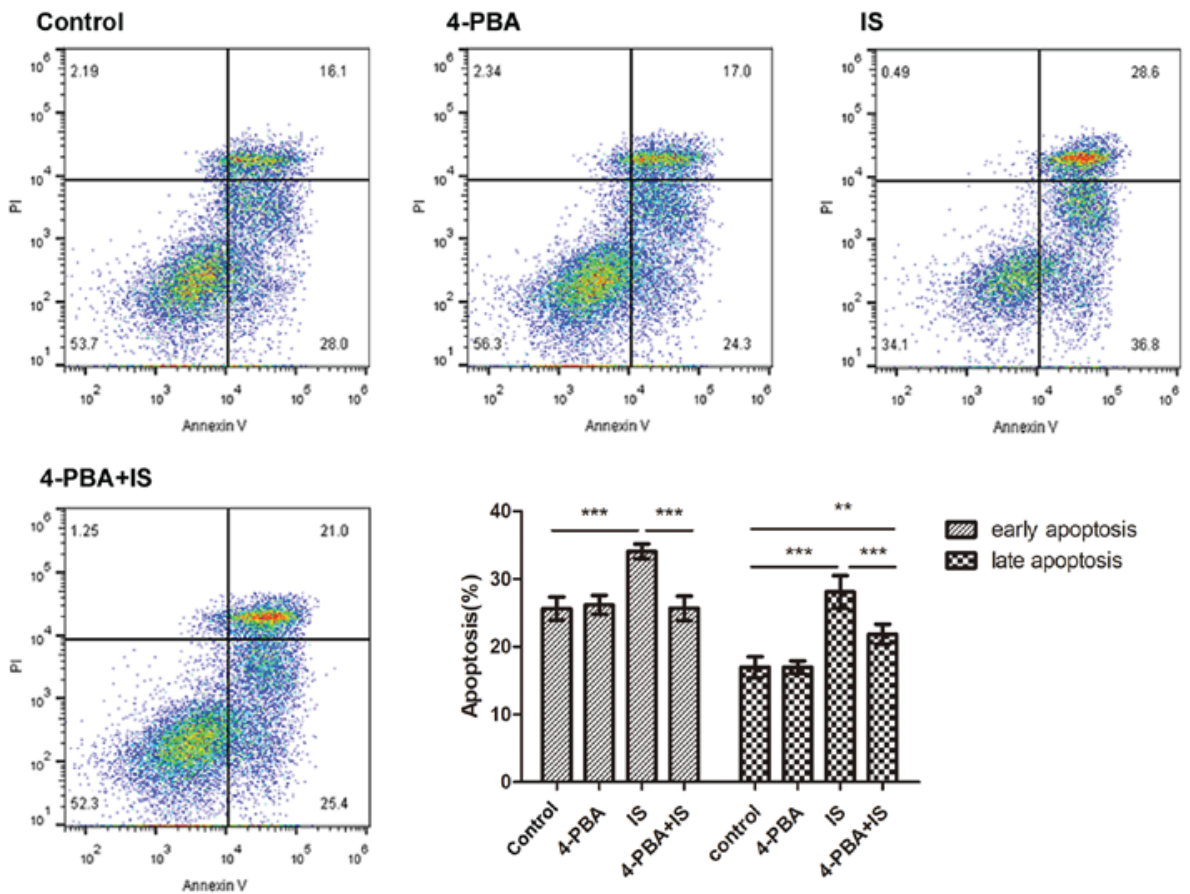

Figure 3. Determination of apoptosis in $\mathrm{H} 9 \mathrm{C} 2$ cells under different treatment conditions. The apoptosis rate of $\mathrm{H} 9 \mathrm{C} 2$ cardiomyocytes in groups 1 to 4 (control, 4-PBA, IS, and 4-PBA+IS) was determined $(\mathrm{n}=4) .{ }^{* *} \mathrm{P}<0.01,{ }^{* * * *} \mathrm{P}<0.001 \mathrm{IS}$, indoxyl sulfate; PI, propidium sulfide; 4-PBA, 4-phenylbutyric acid.
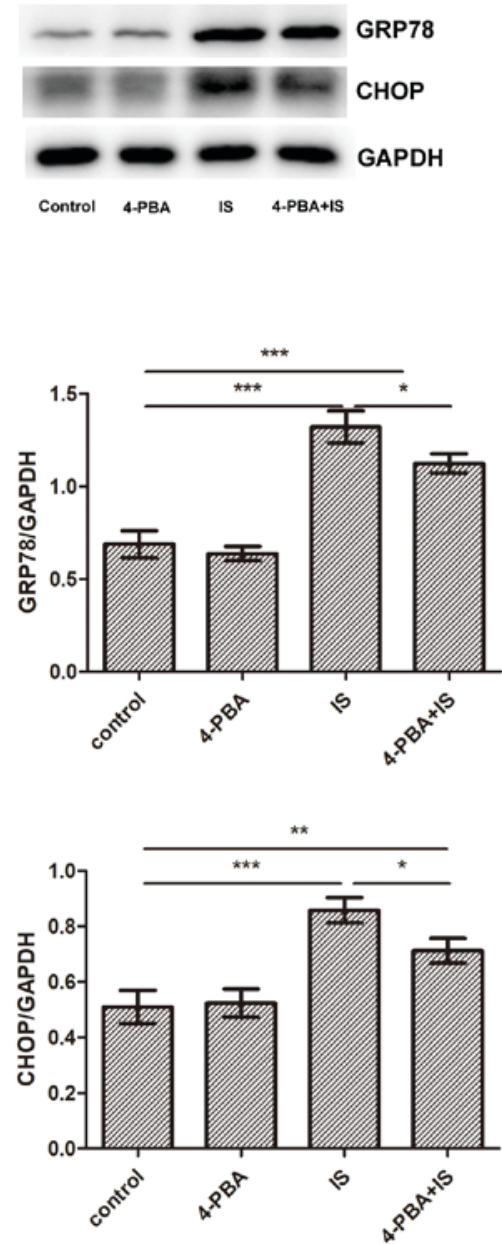

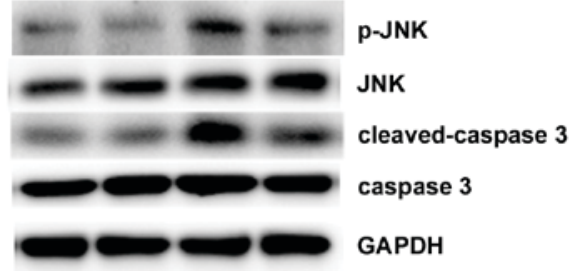

Control 4-PBA IS 4-PBA+1S
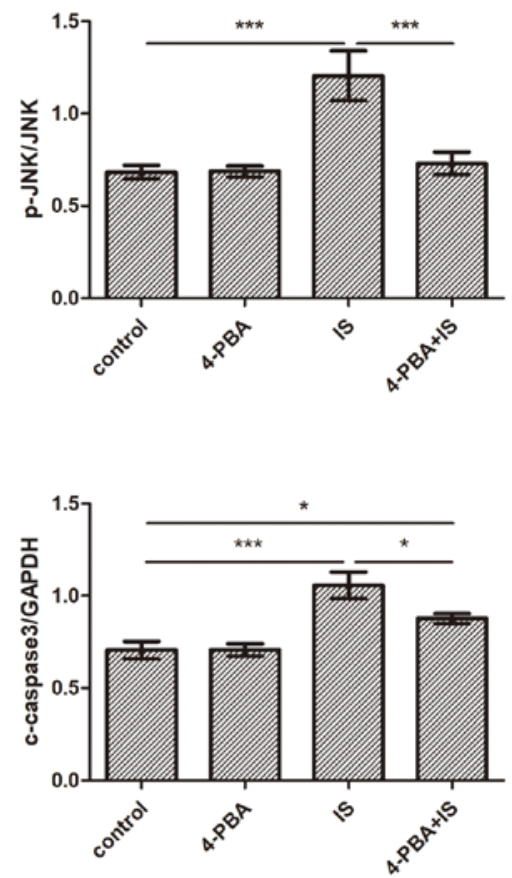

Figure 4. Protein expression levels of GRP78, CHOP, p-JNK and cleaved-caspase 3. The protein expression levels of GRP78, CHOP, p-JNK and cleaved-caspase 3 were determined in $\mathrm{H} 9 \mathrm{C} 2$ cardiomyocytes in groups 1 to 4 (control, 4-PBA, IS, and 4-PBA+IS) (n=4). ${ }^{*} \mathrm{P}<0.05,{ }^{* *} \mathrm{P}<0.01,{ }^{* * * *} \mathrm{P}<0.001$. IS, indoxyl sulfate; 4-PBA, 4-phenylbutyric acid; GRP78, glucose-regulated protein 78; p, phosphorylated; CHOP, CCAAT-enhancer-binding protein homologous protein; JNK, c-Jun N-terminal kinase. 
the CHOP and JNK pathways may be associated with IS-induced ERS and apoptosis. The results identified ERS-related apoptosis as a novel mechanism in IS-induced cardiomyocyte toxicity, providing a potentially valuable therapeutic avenue for the treatment of IS-induced cardiovascular diseases.

However, various limitations of the present study should be mentioned. It is difficult to accurately recapitulate the conditions of CKD in vitro, as CKD is a long-term and complicated pathological progress, and the authors only treated cells with IS for $24 \mathrm{~h}$ in the present study. In addition, the concentration of IS used in the study is higher than would be expected in clinical circumstances, as a low level of IS would not cause damage in cardiomyocytes in the timeframe of the experiment. The authors intend to establish an animal model to further investigate the role of IS in uremic cardiomyopathy and its mechanism, particularly the effect on apoptosis and ERS.

In conclusion, the results suggested that IS, a protein-bound uremic toxin, induces ERS-related apoptosis in H9C2 cardiomyocytes via the CHOP and JNK pathways, and the effects were attenuated by the ERS modulator 4-PBA. The findings will aid the further understanding of the mechanisms of IS cardiotoxicity. As it is difficult to remove IS with current dialysis techniques, further findings regarding the mechanism may contribute to the development of a novel therapeutic strategy, particularly focused on ERS and apoptosis reduction.

\section{Acknowledgements}

Not applicable.

\section{Funding}

The present study was supported by the Science and Technology Commission of Shanghai Municipal (grant no. 14DZ2260200, the project of Shanghai Key Laboratory of Kidney and Blood Purification); Shanghai Committee of Science and Technology, China (grant no. 15DZ0503402); and the Shanghai Municipal Commission of Health and Family Planning (grant no. 2014 ZY3-CCCX-3-3043).

\section{Availability of data and materials}

The datasets used and/or analyzed during the current study are available from the corresponding author on reasonable request.

\section{Authors' contributions}

XT and XSC performed the western blotting and flow cytometry experiments. PZ contributed to the cell culture. FFX and JT participated in experiment design and edited the manuscript. JZZ and XQD designed the present study.

\section{Ethics approval and consent to participate}

Not applicable.

\section{Patient consent for publication}

Not applicable.

\section{Competing interests}

The authors declare that they have no competing interests.

\section{References}

1. Herzog CA, Asinger RW, Berger AK, Charytan DM, Diez J, Hart RG, Eckardt KU, Kasiske BL, McCullough PA, Passman RS, et al: Cardiovascular disease in chronic kidney disease. A clinical update from Kidney Disease: Improving global outcomes (KDIGO). Kidney Int 80: 572-586, 2011.

2. Granata A, Clementi A, Virzi GM, Brocca A, de Cal M, Scarfia VR, Zanoli L, Ronco C, Corrao S and Malatino L: Cardiorenal syndrome type 4: From chronic kidney disease to cardiovascular impairment. Eur J Intern Med 30: 1-6, 2016.

3. Tan X, Cao X, Zou J, Shen B, Zhang X, Liu Z, Lv W, Teng J and Ding X: Indoxyl sulfate, a valuable biomarker in chronic kidney disease and dialysis. Hemodial Int 21: 161-167, 2017.

4. Cao XS, Chen J, Zou JZ, Zhong YH, Teng J, Ji J, Chen ZW, Liu ZH, Shen B, Nie YX, et al: Association of indoxyl sulfate with heart failure among patients on hemodialysis. Clin J Am Soc Nephrol 10: 111-119, 2015.

5. Yisireyili M, Shimizu H, Saito S, Enomoto A, Nishijima F and Niwa T: Indoxyl sulfate promotes cardiac fibrosis with enhanced oxidative stress in hypertensive rats. Life Sci 92: 1180-1185, 2013.

6. Lin CY, Hsu YJ, Hsu SC, Chen Y, Lee HS, Lin SH, Huang SM, Tsai CS and Shih CC: CB1 cannabinoid receptor antagonist attenuates left ventricular hypertrophy and Akt-mediated cardiac fibrosis in experimental uremia. J Mol Cell Cardiol 85: 249-261, 2015.

7. Rani S, Sreenivasaiah PK, Cho C and Kim DH: Salubrinal alleviates pressure overload-induced cardiac hypertrophy by inhibiting endoplasmic reticulum stress pathway. Mol Cells 40: 66-72, 2017.

8. Lu WW, Zhao L, Zhang JS, Hou YL, Yu YR, Jia MZ, Tang CS and Qi YF: Intermedin1-53 protects against cardiac hypertrophy by inhibiting endoplasmic reticulum stress via activating AMP-activated protein kinase. J Hypertens 33: 1676-1687, 2015.

9. Logue SE, Cleary P, Saveljeva S and Samali A: New directions in ER stress-induced cell death. Apoptosis 18: 537-546, 2013.

10. Yoshida H: ER stress and diseases. FEBS J 274: 630-658, 2007.

11. Gao Y, Jia P, Shu W and Jia D: The protective effect of lycopene on hypoxia/reoxygenation-induced endoplasmic reticulum stress in H9C2 cardiomyocytes. Eur J Pharmacol 774: 71-79, 2016.

12. Taddei S, Nami R, Bruno RM, Quatrini I and Nuti R: Hypertension, left ventricular hypertrophy and chronic kidney disease. Heart Fail Rev 16: 615-620, 2011.

13. Siedlecki AM, Jin X and Muslin AJ: Uremic cardiac hypertrophy is reversed by rapamycin but not by lowering of blood pressure. Kidney Int 75: 800-808, 2009.

14. Yang K, Wang C, Nie L, Zhao X, Gu J, Guan X: Klotho protects against indoxyl sulphate-induced myocardial hypertrophy. J Am Soc Nephrol 26: 2434-2446, 2015.

15. Adijiang A, Goto $\mathrm{S}$, Uramoto $\mathrm{S}$, Nishijima $\mathrm{F}$ and Niwa $\mathrm{T}$ : Indoxyl sulphate promotes aortic calcification with expression of osteoblast-specific proteins in hypertensive rats. Nephrol Dial Transplant 23: 1892-1901, 2008.

16. Yang K, Xu X, Nie L, Xiao T, Guan X, He T, Yu Y, Liu L, Huang Y, Zhang $\mathbf{J}$ and Zhao J: Indoxyl sulfate induces oxidative stress and hypertrophy in cardiomyocytes by inhibiting the AMPK/UCP2 signaling pathway. Toxicol Lett 234: 110-119, 2015.

17. Lin CJ, Liu HL, Pan CF, Chuang CK, Jayakumar T, Wang TJ, Chen $\mathrm{HH}$ and $\mathrm{Wu} \mathrm{CJ}$ : Indoxyl sulfate predicts cardiovascular disease and renal function deterioration in advanced chronic kidney disease. Arch Med Res 43: 451-456, 2012.

18. Yu M, Kim YJ and Kang DH: Indoxyl sulfate-induced endothelial dysfunction in patients with chronic kidney disease via an induction of oxidative stress. Clin J Am Soc Nephrol 6: 30-39, 2011.

19. Lin CJ, Pan CF, Liu HL, Chuang CK, Jayakumar T and Wang TJ: The role of protein-bound uremic toxins on peripheral artery disease and vascular access failure in patients on hemodialysis. Atherosclerosis 225: 173-179, 2012.

20. Fujii H, Nishijima F, Goto S, Sugano M, Yamato H and Kitazawa R, Kitazawa S and Fukagawa M: Oral charcoal adsorbent (AST-120) prevents progression of cardiac damage in chronic kidney disease through suppression of oxidative stress. Nephrol Dial Transplant 24: 2089-2095, 2009. 
21. Zhang K and Kaufman RJ: From endoplasmic-reticulum stress to the inflammatory response. Nature 454: 455-462, 2008.

22. Kupsco A and Schlenk D: Oxidative stress, unfolded protein response, and apoptosis in developmental toxicity. Int Rev Cell Mol Biol 317: 1-66, 2015.

23. Wang WJ, Cheng MH, Sun MF, Hsu SF and Weng CS: Indoxyl sulfate induces renin release and apoptosis of kidney mesangial cells. J Toxicol Sci 39: 637-643, 2014

24. Ellis RJ, Small DM, Ng KL, Vesey DA, Vitetta L and Francis RS: Indoxyl sulfate induces apoptosis and hypertrophy in human kidney proximal tubular cells. Toxicol Pathol 46: 449-459, 2018.
25. Kim SH, Yu MA, Ryu ES, Jang YH and Kang DH: Indoxyl sulfate-induced epithelial-to-mesenchymal transition and apoptosis of renal tubular cells as novel mechanisms of progression of renal disease. Lab Invest 92: 488-498, 2012.

26. Kim YH, Kwak KA, Gil HW, Song HY and Hong SY: Indoxyl sulfate promotes apoptosis in cultured osteoblast cells. BMC Pharmacol Toxicol 14: 60, 2013. 